\title{
Summary of HINS Focusing Solenoid Production and Tests
}

\author{
Michael A. Tartaglia, Member IEEE, Earle Burkhardt, Tony Leach, Darryl F. Orris, \\ Roger Rabehl, Iouri Terechkine, and John C. Tompkins
}

\begin{abstract}
A compact R\&D RF linac is being built at Fermilab to study several key technologies related to accelerating high intensity beams. One of the goals is reduction of beam losses through the use of solenoid lenses in the low energy front end of the linac. A total of 23 compact, high field, superconducting solenoids have been procured by Fermilab for the first (roomtemperature RF) section of the linac. In this report we summarize the quench and magnetic performance of the lenses.
\end{abstract}

Index Terms-Superconducting solenoid accelerator magnet

\section{INTRODUCTION}

$\mathrm{T}$ he construction of a proton linac is an ongoing $R \& D$ effort at Fermilab to develop the technologies needed for a high intensity neutrino source (HINS) [1]. Following an extensive research and development program to qualify designs and manufacturing technologies [2], a total of 23 solenoid cold masses were procured for use as focusing elements in the low energy front end of the linac. Of the 23 solenoids, thirteen are magnets without corrector coils that are designated "Type-1", and ten are "Type-2" magnets which have horizontal and vertical dipole coils nested within the solenoid main coil. All of the solenoids have field cancelling "bucking coils" at both ends, in order to attenuate the field in the vicinity of adjacent RF cavities in the beam line. Table I gives an overview of the performance parameters for focusing lenses in this first (room temperature RF) section of the linac.

The industrial vendor Cryomagnetics, Inc. was awarded a contract to supply these magnets after fully testing them in welded stainless steel liquid helium vessels, to demonstrate that they meet specified performance requirements. The lenses will be assembled into individual cryostats at Fermilab [3], and the completed devices will be cold tested again, to measure alignment properties, before installation into the beam line.

Manuscript received 20 October 2009. Work supported by the U.S. Department of Energy under contract No. DE-AC02-07CH11359.

M. A. Tartaglia, D. F. Orris, R. Rabehl, I. Terechkine and J. C. Tompkins are with the Fermi National Accelerator Laboratory, Batavia, IL 60510 USA Corresponding author Michael A. Tartaglia, phone: 630-840-3890; fax: 630840-8079; e-mail: tartaglia@fnal.gov.

E. Burkhardt and T. Leach are with Cryomagnetics, Inc. Oak Ridge, TN 37830 USA.

\section{Production OvervieW}

\section{A. Schedule and Status}

Fabrication started in the spring of 2008 and the first tests by the vendor were conducted in June 2008. As of October 2009 , only three solenoids remain to be completed; all others have been successfully tested and have met the specified requirements.

TABLE I. SOLENOID LENS MAGNETIC PARAMETERS

\begin{tabular}{ccc}
\hline \hline Parameter & Type-1 & Type-2 \\
\hline Focusing Field Integral, $\mathrm{JB}^{2} \mathrm{dL}$ & $180 \mathrm{~T}^{2}-\mathrm{cm}$ & $180 \mathrm{~T}^{2}-\mathrm{cm}$ \\
Measured Field Integral at $180 \mathrm{~A}$ & $168 \mathrm{~T}^{2}-\mathrm{cm}$ & $168 \mathrm{~T}^{2}-\mathrm{cm}$ \\
Measured Fringe Field at $180 \mathrm{~A}$ & $45 \mathrm{G}$ & $90 \mathrm{G}$ \\
\hline Parameter & Horizontal & Vertical \\
Steering Dipole Strength, $\int \mathrm{BdL}$ & $0.25 \mathrm{~T}-\mathrm{cm}$ & $0.25 \mathrm{~T}-\mathrm{cm}$ \\
Dipole Integral Strength at 200 A & $0.92 \mathrm{~T}-\mathrm{cm}$ & $0.88 \mathrm{~T}-\mathrm{cm}$ \\
\hline \hline
\end{tabular}

\section{B. Test Requirements}

All solenoids and steering dipoles were required to pass hipot tests to ensure insulation integrity to withstand voltage excursions during quench. The coils were tested at $1000 \mathrm{~V}$ in air, and again at $500 \mathrm{~V}$ in liquid helium, to demonstrate that leakage current to ground (the steel vessel) was less than 1 $\mu \mathrm{A}$. One solenoid initially failed this test, due to pinched insulation of a lead during assembly; it did pass the hi-pot after repair.

The expected maximum quench current of the solenoids was predicted from the superconductor strand short sample characteristics, critical current Ic versus field, measured at 4.2 $\mathrm{K}$ at Fermilab's Short Sample Test Facility. The load-lines at peak field points in each coil were determined using magnetic models of the as-designed solenoids, and predictions were validated by tests of prototype solenoids at Fermilab [2]. The vendor was asked to train each solenoid at low $(1 \mathrm{~A} / \mathrm{s}) \mathrm{ramp}$ rate until it achieved three quenches above $210 \mathrm{~A}, 85 \%$ of the predicted maximum "short sample" current (after the first solenoid reached this level without quenching). During vendor tests, no energy extraction system was used and the stored energy was dissipated within the magnet coils.

Dipole coils, made from the same strand as the solenoid main coil, run at low current; they have a weak self field and generous operating margin, and are not expected to quench 
even in the solenoid field. Therefore they were required to reach $250 \mathrm{~A}$ in the field of the solenoid at $180 \mathrm{~A}$, the nominal operating current.

A Hall probe position scan of the axial magnetic field was required for each solenoid at the nominal operating current, $180 \mathrm{~A}$, to capture the field peak strength and longitudinal profile, which characterizes the focusing strength. Also measured in this scan was the field strength in the fringe region, $\pm 150 \mathrm{~mm}$ from the solenoid center; if the bucking coil geometry is correct, this must be less than a specified level. In addition to this scan, measurements of the peak axial field were made as a function of the current going up and then down, to examine the behavior of superconductor magnetization and iron saturation. A position scan of each steering dipole was made at 200 A to check the transverse field shape and integral strength.

\section{Quality Assurance}

After review and approval of the vendor test results, magnets were shipped to Fermilab. Mechanical dimensions were inspected against tolerances, helium vessels were leak checked and electrical hi-pot tests were performed. All magnets passed these inspections with only a few minor issues that were easily resolved. Prior to assembling each solenoid into a cryostat, an additional magnetic field polarity check is made and dipole field angles are measured using a magnetometer-based device.

A subset of the magnets was re-tested at Fermilab in liquid helium as a quality assurance measure, to validate vendor procedures and results, cross-calibrate current and field values, study quench performance after a thermal cycle, and investigate any anomalous behavior. The first four production magnets - two of each type - were re-tested and the final test procedures converged. Subsequently one solenoid from each batch of four was selected for re-testing. A total of seven solenoids have been re-tested at Fermilab, with two more tests pending. Fig. 1 shows a photo of a production solenoid being assembled into a cryostat at Fermilab.

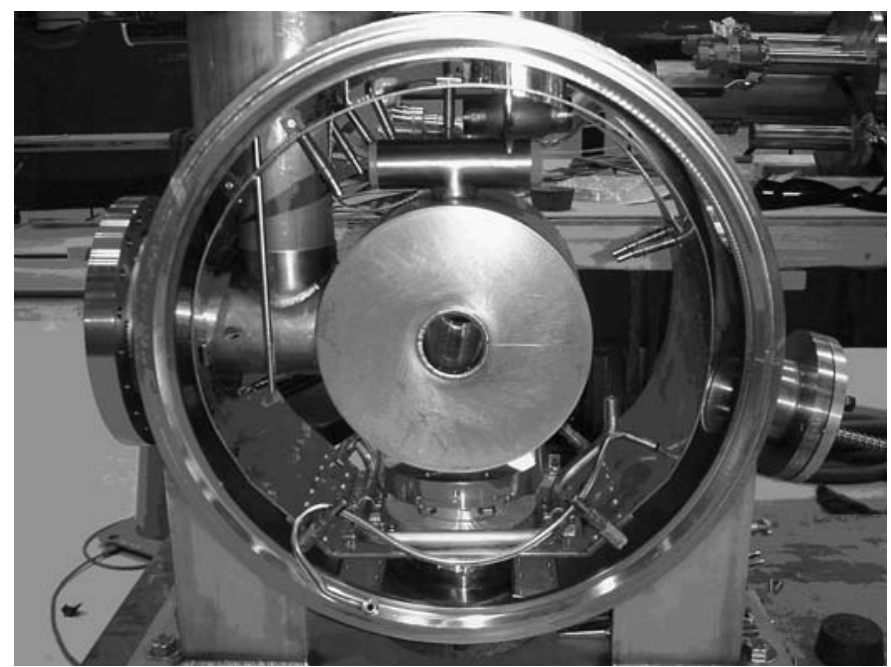

Fig. 1. Photo of the first Type-2 solenoid in its helium vessel being assembled into a cryostat at Fermilab.

\section{Test REsults}

\section{A. Solenoid Quench Performance}

The vendor quench training was performed at $4.2 \mathrm{~K}$ in boiling liquid helium. Training curves are shown in Fig. 2 for all of the Type- 1 solenoids, and Fig. 3 for the Type-2 solenoids. Not all solenoids reached their maximum current within the required three quenches above the 210 A threshold, but most solenoids reached the expected maximum current with 5 or 6 quenches.

One Type-2 solenoid (T2_09) showed significantly slower training than the others: many of these quenches were located in the bucking coils, which absorb all of the stored energy during a quench and heat up significantly (especially when no energy extraction circuit is utilized). Thus, one contributing factor to the slow training may be that insufficient time was allowed between ramps for the bucking coil temperature to equilibrate with the helium bath. A re-test of this device at Fermilab is planned, to continue to explore its training behavior after a thermal cycle.

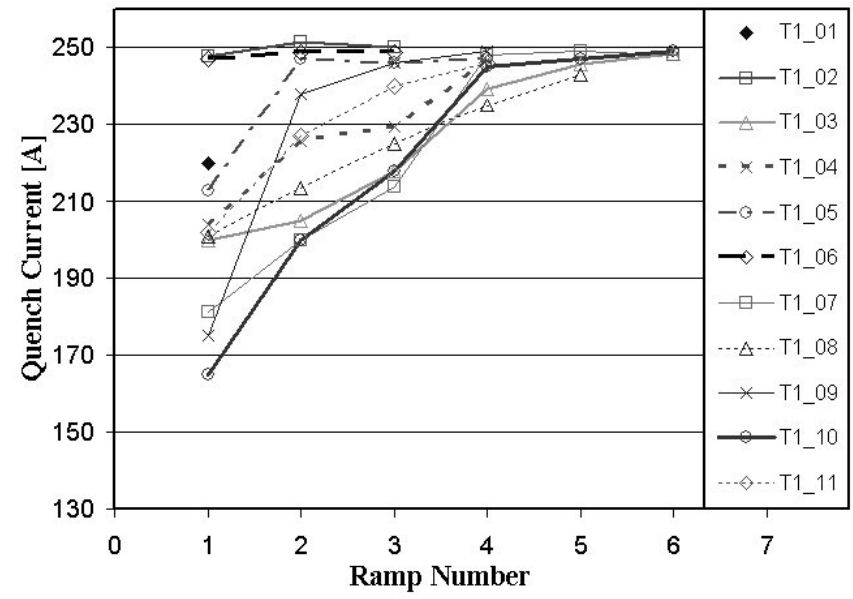

Fig. 2. Type-1 Solenoid quench training by the vendor at $4.2 \mathrm{~K}$. Filled symbol indicates maximum current with no quench. Expected maximum current is $250 \mathrm{~A}$.

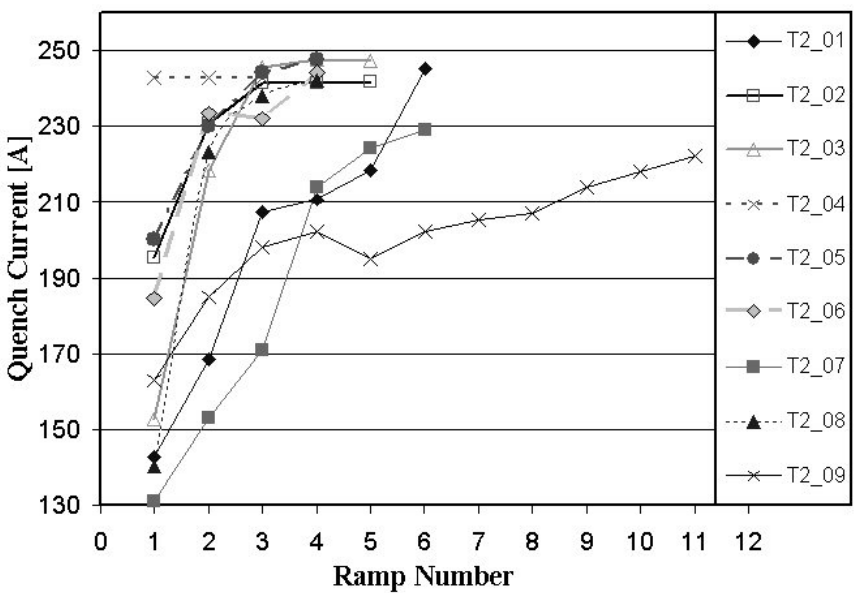

Fig. 3. Type-2 Solenoid quench training by the vendor at $4.2 \mathrm{~K}$. Expected maximum current is $245 \mathrm{~A}$. 
Fig. 4 shows the re-tested solenoid training curves at a slightly higher temperature, $4.43 \mathrm{~K}$ (due to slight overpressure in a sealed test dewar), for both Type-1 and Type-2. All solenoids appear to re-train very quickly, reaching the expected maximum current in at most three quenches. In each case the first quench was above the nominal operating current.

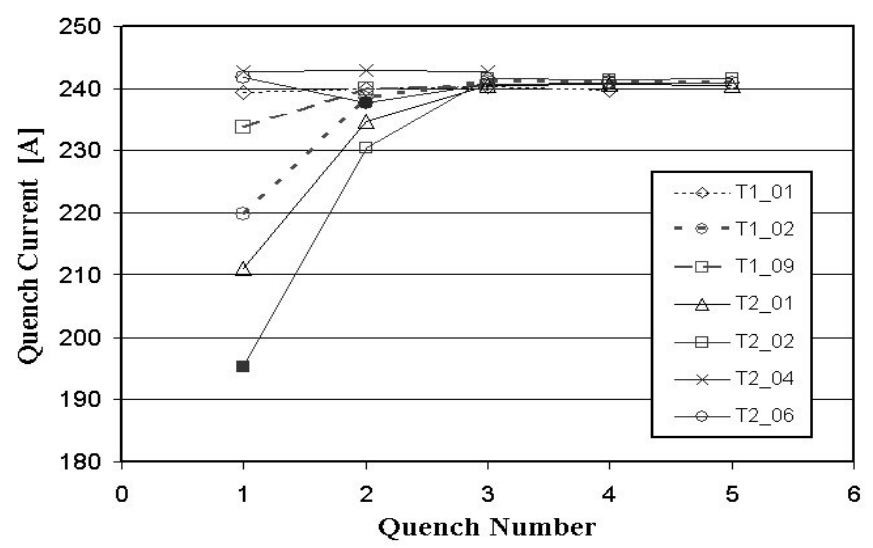

Fig. 4. Type- 1 and Type- 2 quench re-training at Fermilab at $4.43 \mathrm{~K}$. Open symbols are Main Coil, Filled symbols are Bucking Coil quenches.

\section{B. Steering Dipole Quench Performance}

As expected, due to the large operating margin, all dipole coils reached the required current level in the solenoid field without quenching in tests performed by the vendor and when re-tested at Fermilab.

\section{Solenoid Magnetic Performance}

Fig. 5 shows an overlay of all Type-1 solenoid axial field transfer function profiles measured at the nominal operating current by the manufacturer. The shapes are clearly very symmetric and consistent.

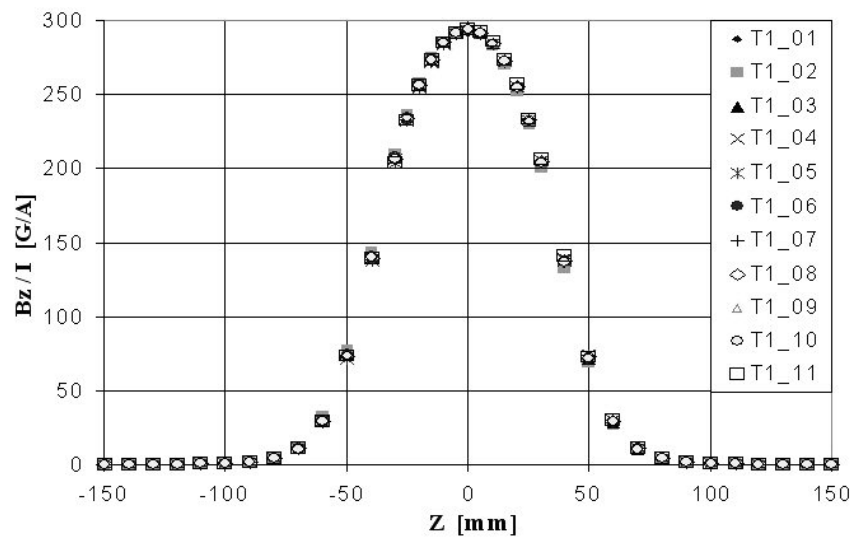

Fig. 5. Vendor-measured Type-1 axial field profiles at 180 A nominal operating current.

The results of "stair-step" up and down measurements at the peak field position $(\mathrm{Z}=0)$ are shown in Fig. 6 for the Type-1 solenoids. Measurements were taken on the up-going current ramp at $10,100,180,200$ and $210 \mathrm{~A}$, then again at $10 \mathrm{~A}$ on the down ramp. These data show some saturation at high current, which is consistent with expectations from prototype tests and the prediction of a Vector Fields magnetic model. They also exhibit hysteretic behavior which will be discussed next. The variation in peak strength is less than $1 \%$ for the ensemble. Type-2 magnets show similar behavior and consistency (although with a different peak strength due to the difference in coil geometry necessary for the nested dipole coils). The agreement of absolute strength with Fermilab measurements is also within $1 \%$ for both Type- 1 and Type- 2 solenoids.

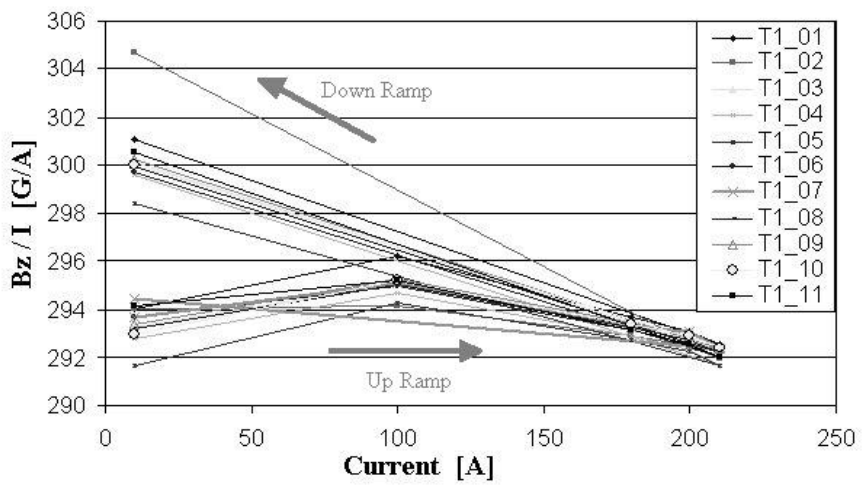

Fig. 6. Vendor-measured Type-1 peak transfer function versus current (stairstep up, down). Type-2 behavior and consistency are similar.

The vendor measurements of fringe field at $\pm 150 \mathrm{~mm}$ from the center showed some unexpected variation that led to further study during the re-tests at Fermilab. In particular, some solenoids showed fringe field levels that were much lower than the others. Several of these magnets were re-tested at Fermilab, where the fringe field levels were all found to be very consistent, as can be seen for the re-tested Type-2 solenoid profiles in Fig. 7.

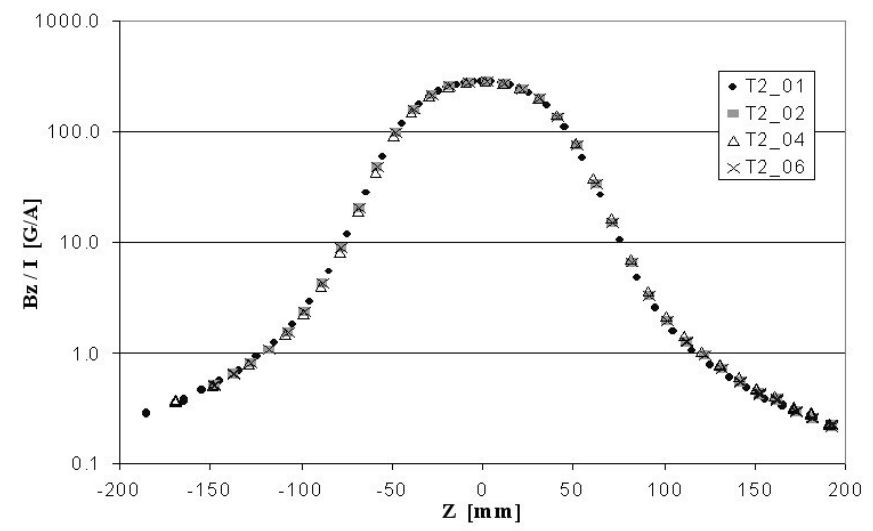

Fig. 7. Fermilab-measured Type- 2 axial field profiles at the 180 A nominal operating current. Note the log scale and consistency of fringe field levels.

The solution to this puzzle is related to the hysteresis behavior, and the way in which the measurements were made. First, the hysteresis is caused by magnetization of the superconductor strands during a ramp cycle: Fig. 8 shows the resulting field measured at $0 \mathrm{~A}$ current following a ramp up and down. Second, the vendor's procedure was to locate the center of the solenoid and zero the Hall probe readout at that point prior to performing the magnetic field scans: because of the magnetization field, this effectively subtracts an offset field of up to 60 Gauss. This explains the variation in fringe field results, and has virtually no impact on the peak strength or field integral. At Fermilab, Hall probe offsets were 
determined well outside the solenoid, before powering the magnet (so, the Earth field may contribute at the level of $1 \mathrm{G}$ ).

Interestingly, several measurements similar to Fig. 8 were made after quenches and show that this field is partially or fully removed (presumably depending upon the details of the quench development in the various coils). Thus, we conclude that the iron flux return does not to contribute to the hysteresis - as was expected, since the "soft" iron material (1008/1010 low carbon steel) was chosen for this property.

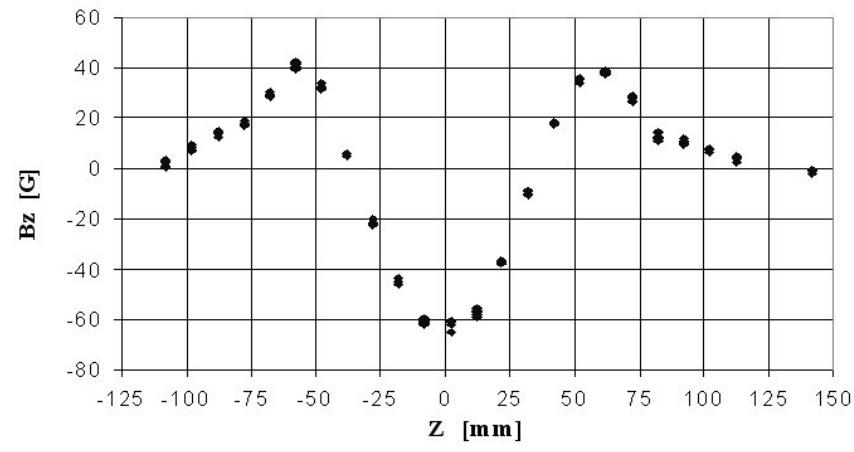

Fig. 8. Superconductor magnetization field after ramp up to $225 \mathrm{~A}$ and down to $0 \mathrm{~A}$ again. The peak field direction is the same as the powered solenoid field direction. The positive field excursions are caused by the bucking coils.

\section{Steering Dipole Magnetic Performance}

The steering dipole coils are each wound in a single layer on a G10 form, with the horizontal dipole (HD) nested within the vertical dipole (VD). The transverse field profiles for the steering coils are shown in Fig. 9 and Fig. 10, respectively for the VD and HD correctors, as measured at $200 \mathrm{~A}$. The required bending field integral of $0.25 \mathrm{~T}-\mathrm{cm}$ is nominally reached at $50 \mathrm{~A}$, but measuring them at the higher current gives greater precision. The field profiles are very consistent (HD and VD shapes differ slightly because of the different coil geometry). The fractional variation in peak strengths is small: $1.9 \%$ for $\mathrm{VD}$, and $1.2 \%$ for $\mathrm{HD}$. In corresponding measurements at Fermilab, the peak field strengths were found to be lower by about $5 \%$. Further study of the transverse Hall probe calibrations will be made to understand this difference. However, from a practical standpoint, this small discrepancy will not impact the use of these weak steering correction coils.

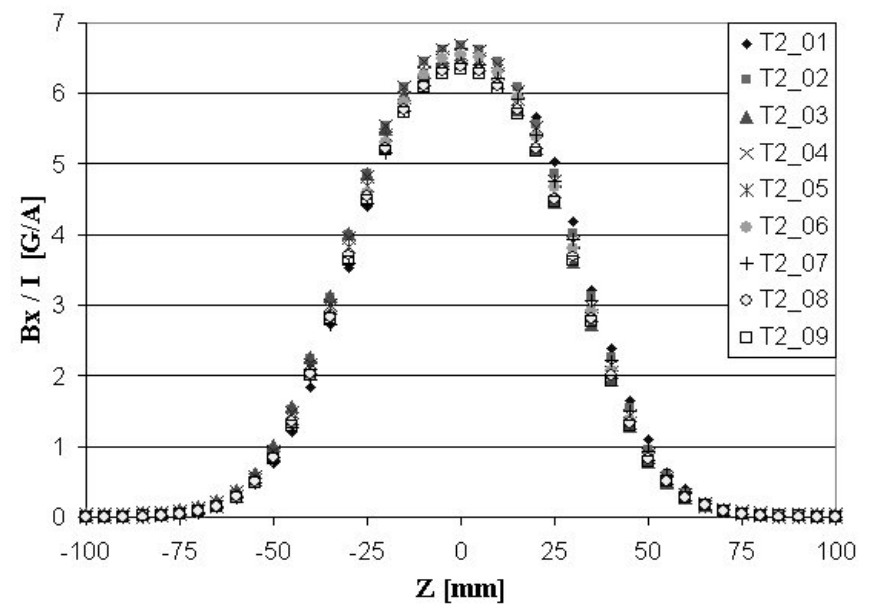

Fig. 9. Ensemble of Vertical Dipole transverse field profiles measured by the vendor.

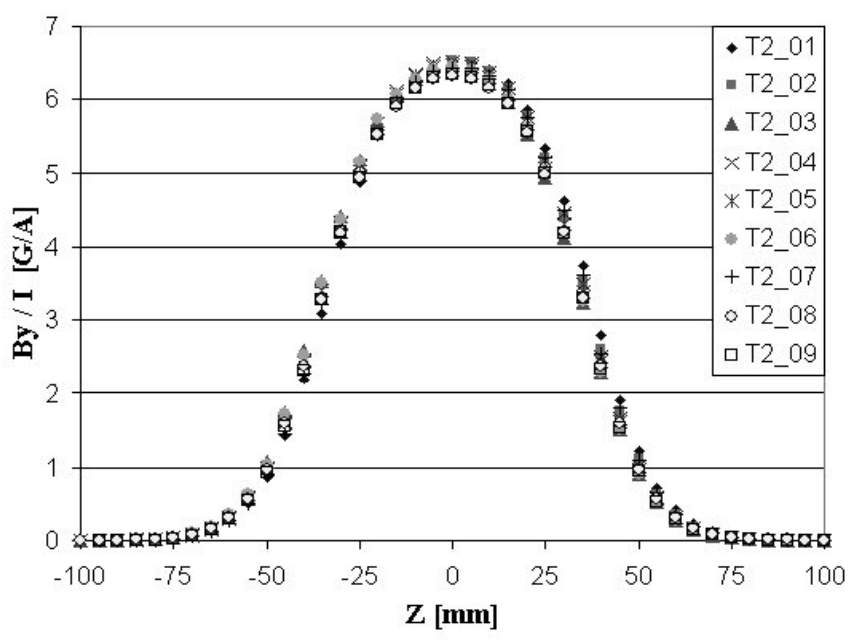

Fig. 10. Ensemble of Horizontal Dipole transverse field profiles measured by the vendor.

\section{SUMMARY}

An industrial supplier was awarded the contract to build, test, and deliver twenty-three strong superconducting solenoids for the HINS R\&D proton linac at Fermilab, ten Type-2 magnets with dipole steering correctors, and thirteen Type-1 magnets without correctors. The cold masses in their liquid helium vessels were all to be quench tested, and their magnetic fields to be mapped, by the supplier. A subset of the production magnets, about one in four, was re-tested by Fermilab as a quality assurance measure.

Twenty cold masses have now been built, tested, and delivered, and the final three should soon be complete. All twenty magnets meet the required performance specifications. Hi-pot tests show no insulation problems at $1000 \mathrm{~V}$ in air and $500 \mathrm{~V}$ in liquid helium. They exhibit reasonably fast quench training, and very little re-training is seen in those re-tested solenoids. The steering dipoles required no training quenches to reach a current five times higher than their nominal operating point. The magnetic field profiles and peak field transfer functions for all of the solenoids and steering dipoles show very good consistency. The specified low fringe field levels have all been achieved.

\section{ACKNOWLEDGMENT}

The authors would like to thank the staff members at Cryomagnetics, Inc. and at Fermilab for their diverse and skillful contributions to the design, procurement, fabrication, testing, shipment, and handling of these devices.

\section{REFERENCES}

[1] G. Apollinari, et al., "HINS Linac Front End Focusing System R\&D," IEEE Trans. Appl. Supercon., Vol. 19, No. 3, June 2009, pp.1368-1371.

[2] M. Tartaglia, et al., "Test Results for HINS Focusing Solenoids at Fermilab," IEEE Trans. Appl. Supercon., Vol. 19, No. 3, June 2009, pp.1352-1355.

[3] T. Page, et al., "HINS Superconducting Lens and Cryostat Performance," IEEE Trans. Appl. Supercon., Vol. 19, No. 3, June 2009, pp.1356-13 